\title{
Atmosfera refrigerada e controlada para pêssegos 'Eragil'
}

\author{
Cold and controlled atmosphere of 'Eragil' peaches
}

\author{
Auri Brackmann ${ }^{\mathrm{I} *}$ Elizandra Pivotto Pavanello ${ }^{\mathrm{I}}$ Vanderlei Both ${ }^{\mathrm{I}}$ Anderson Weber ${ }^{\mathrm{I}}$ \\ Josuel Alfredo Vilela Pinto ${ }^{\mathrm{I}}$
}

\section{RESUMO}

O objetivo do trabalho foi avaliar as condições de atmosfera controlada em associação à baixa temperatura, visando a manter a qualidade e diminuir as perdas durante o armazenamento de pêssegos da cultivar 'Eragil'. Os frutos foram conservados nas seguintes condições: atmosfera refrigerada, atmosfera controlada com pressões parciais de 1,0kPa de $\mathrm{O}_{2}+3,0 \mathrm{kPa}$ de $\mathrm{CO}_{2}, 2,0 \mathrm{kPa}$ de $\mathrm{O}_{2}+5,0 \mathrm{kPa}$ de $\mathrm{CO}_{2}$, 2,0kPa de $\mathrm{O}_{2}+8,0 \mathrm{kPa}$ de $\mathrm{CO}_{2}$, sendo todos armazenados por um período de 57 dias, a $-0,5^{\circ} \mathrm{C}$. O delineamento experimental utilizado foi o inteiramente casualizado, com oito repetições de 10 frutos. Após 57 dias de armazenamento e dois dias de exposição a $20^{\circ} \mathrm{C}$, os frutos em atmosfera controlada mantiveram maior acidez titulável e apresentaram menor incidencia de escurecimento interno da polpa. Os sólidos solúveis e a taxa respiratória não apresentaram diferença entre as condições de armazenamento. Entretanto, todas as condições de atmosfera controlada apresentaram maior síntese de etileno $e$ atividade ACC oxidase em relação ao armazenamento em atmosfera refrigerada. Após a exposição à temperatura de $20^{\circ} \mathrm{C}$, os frutos armazenados em 2,0kPa de $\mathrm{O}_{2}+8,0 \mathrm{kPa}$ de $\mathrm{CO}_{2}$ apresentaram maior degradação da firmeza, menor incidência de lanosidade, escurecimento da polpa e da epiderme, sendo esta a condição de atmosfera controlada mais eficiente para o armazenamento de pêssegos 'Eragil'.

Palavras-chave: Prunus persica L., pós-colheita, escurecimento interno, lanosidade, qualidade.

\section{ABSTRACT}

The objective of this research was to evaluate the conditions of controlled atmosphere associated with low temperature, aiming to maintain the quality and reduce losses during storage of 'Eragil' peaches. The evaluated treatments were cold atmosfhere, controlled atmosphere (CA) with partial pressure of $1.0 \mathrm{kPa}$ of $\mathrm{O}_{2}+3.0 \mathrm{kPa}$ of $\mathrm{CO}_{2}, 2.0 \mathrm{kPa}$ of $\mathrm{O}_{2}+$ $5.0 \mathrm{kPa}$ of $\mathrm{CO}_{2}$ and $2.0 \mathrm{kPa}$ of $\mathrm{O}_{2}+8.0 \mathrm{kPa}$ of $\mathrm{CO}_{2}$, all stored for a period of 57 days at $-0.5^{\circ} \mathrm{C}$. The experimental design was completely randomized with eight repetitions. After 57 days of storage plus two days of exposure to $20^{\circ} \mathrm{C}$, fruits in controlled atmosphere maintained higher acidity, and showed less damage caused by internal flesh browning. The soluble solids, skin browning, rot and respiration rate showed no difference between the storage conditions, however all CA conditions had higher synthesis of ethylene and ACC oxidase activity. After shelf life, fruits stored in $2.0 \mathrm{kPa}$ de $\mathrm{O}_{2}+8.0 \mathrm{kPa}$ de $\mathrm{CO}_{2}$ showed higher firmness loss, lower incidence of woolliness, internal and skin browning, and therefore the most effective CA condition for 'Eragil' peaches storage.

Key words: Prunus persica L., postharvest, internal browning, woolliness, quality.

\section{INTRODUÇÃO}

O pêssego 'Eragil’ é considerado de duplo propósito, podendo ser destinado à indústria e ao consumo in natura. Apresenta polpa amarela e sem aderência ao caroço, de tamanho grande, formato oblongo, com aproximadamente $50 \%$ de coloração externa avermelhada.

Pelo fato de ser um pêssego de alta qualidade organoléptica e maturação tardia, apresenta uma boa aceitação comercial e sua produção vem aumentando significativamente. A alta perecibilidade e o curto período de colheita dos pêssegos de mesa exigem o armazenamento de parte da produção para prolongar o período de oferta. Por ser uma cultivar nova, em início de expansão no país, é necessário o estudo de técnicas eficientes para prolongar o período de

IDepartamento de Fitotecnia, Universidade Federal de Santa Maria (UFSM), 97105 900, Santa Maria, RS, Brasil. E-mail: auribrackmann@gmail.com.*Autor para correspondência. 
armazenamento reduzindo a perda das características organolépticas do fruto decorrente da maturação, da ocorrência de distúrbios fisiológicos e das podridões.

A temperatura é um fator importante na manutenção da qualidade em pêssegos. Segundo HADLICH \& ARAÚJO (1993), a baixa temperatura retarda a perda da firmeza e o escurecimento interno da polpa, além de evitar o aparecimento da lanosidade. Nesse sentido, a temperatura mais adequada, para o armazenamento de pêssegos, tem sido apontada como $-0,5^{\circ} \mathrm{C}$ (CERETTAet al., 2000; NAVA\& BRACKMANN, 2002). Além disso, o uso da atmosfera controlada (AC), por meio da redução dos níveis de $\mathrm{O}_{2}$, e a elevação dos níveis de $\mathrm{CO}_{2}$ podem prolongar a qualidade póscolheita dos frutos, diminuir o metabolismo de pigmentos, compostos fenólicos e voláteis (BEAUDRY, 1999) e reduzir a síntese e a ação do etileno sobre o metabolismo dos frutos e a ocorrência de podridões (BRACKMANN \& CHITARRA, 1998). Segundo LURIE \& CRISOSTO (2005), dependendo da cultivar, da temperatura e do tempo de armazenamento, o aumento no nível do $\mathrm{CO}_{2}$ é favorável para a redução de desordens fisiológicas e, segundo AGAR et al. (1990), o alto $\mathrm{CO}_{2}$ e as baixas temperaturas reduzem o desenvolvimento fúngico.

Para que o processo de conservação dos produtos vegetais seja eficiente, é importante se determinarem as pressões parciais de $\mathrm{O}_{2}$ e $\mathrm{CO}_{2}$ mais adequadas, pois estas afetam a respiração (MATHOOKO, 1996) e a síntese de etileno dos frutos (MATHOOKO, 2001). A elevação dos níveis de $\mathrm{CO}_{2}$ (520\%) promove uma inibição da produção de etileno, devido à inibição da atividade da ACC sintase e da ACC oxidase (MATHOOKO, 2001). Os níveis de $\mathrm{O}_{2}$ inferiores a $8 \%$ reduzem significativamente a biossíntese do etileno (KADER, 1986). Além disso, devido ao $\mathrm{O}_{2}$ ser substrato na reação catalisada pela ACC oxidase, sob condições de anaerobiose, a conversão de ACC a etileno pode ser completamente inibida (KENDE, 1993). No entanto, pressões parciais de $\mathrm{O}_{2}$ muito baixas ou altas de $\mathrm{CO}_{2}$ podem induzir a respiração anaeróbica, formando compostos tóxicos que podem desencadear distúrbios fisiológicos e gosto alcoólico (SAQUET et al., 2000).

As pressões parciais de $\mathrm{O}_{2}$ e $\mathrm{CO}_{2}$ durante o armazenamento de pêssegos 'Eragil' ainda não foram estabelecidas, mas, segundo BRACKMANN et al. (2007), na cultivar 'Granada', o uso de AC com pressões parciais de 2,0kPa de $\mathrm{O}_{2}+5,0 \mathrm{kPa}$ de $\mathrm{CO}_{2}$ a $-0,5^{\circ} \mathrm{C}$ diminui consideravelmente a incidência de podridões. Já para a manutenção da firmeza de polpa em pêssegos da cultivar 'Eldorado', CERETTA et al. (2000) determinaram como condição ideal $1,0 \mathrm{kPa}$ de $\mathrm{O}_{2}$ e $3,0 \mathrm{kPa}$ de $\mathrm{CO}_{2}$.
Segundo KADER \& MITCHELL (1989), pêssegos toleram concentrações de $2,0 \mathrm{kPa}$ de $\mathrm{O}_{2}$ e de $5,0 \mathrm{kPa}$ de $\mathrm{CO}_{2}$, enquanto WATADA et al. (1979) considera que $1,0 \mathrm{kPa}$ de $\mathrm{O}_{2}$ e $5 \mathrm{kPa}$ de $\mathrm{CO}_{2}$ mantêm a qualidade e o sabor superiores a AR.

Sendo assim, o objetivo deste trabalho é avaliar as condições ideais de atmosfera controlada em associação à temperatura, visando a diminuir as perdas e manter a qualidade dos frutos durante o armazenamento.

\section{MATERIAL E MÉTODOS}

O trabalho foi conduzido no Núcleo de Pesquisa em Pós-colheita do Departamento de Fitotecnia da Universidade Federal de Santa Maria, no ano de 2007. Os frutos da cultivar 'Eragil' provenientes de um pomar comercial de Bento Gonçalves, Rio Grande do Sul (RS), foram selecionados, descartando os que apresentavam algum tipo de ferimento. Após a seleção, realizou-se a homogeneização das amostras experimentais, que foram armazenadas em minicâmaras experimentais de AC, com capacidade de 233L. O delineamento experimental utilizado foi o inteiramente casualizado, com oito repetições, contendo 10 frutos cada. Os tratamentos avaliados foram: [1] atmosfera refrigerada (AR), [2] AC com 1,0kPa de $\mathrm{O}_{2}+3,0 \mathrm{kPa}$ de $\mathrm{CO}_{2}$; [3] AC com 2,0kPa de $\mathrm{O}_{2}+5,0 \mathrm{kPa}$ de $\mathrm{CO}_{2}$ e [4] AC com 2,0kPa de $\mathrm{O}_{2}+8,0 \mathrm{kPa}$ de $\mathrm{CO}_{2}$, todos armazenados por um período de 57 dias, a $-0,5^{\circ} \mathrm{C}$.

As pressões parciais dos gases $\left(\mathrm{O}_{2}\right.$ e $\left.\mathrm{CO}_{2}\right)$ foram obtidas com a instalação da atmosfera por meio da diluição do $\mathrm{O}_{2}$ nas minicâmaras, com a injeção de $\mathrm{N}_{2}$, obtido por meio de um gerador de nitrogênio com sistema Pressure Swing Adsorption - PSA, seguido por uma injeção de $\mathrm{CO}_{2}$, proveniente de cilindros de alta pressão, até atingir os níveis desejados para cada tratamento. As pressões parciais foram monitoradas e corrigidas diariamente com o uso de analisadores eletrônicos, marca Kronenberger/Climasul. $\mathrm{O} \mathrm{O}_{2}$ consumido durante o processo respiratório dos frutos foi reposto por meio da injeção de ar atmosférico no interior da minicâmaras, e o $\mathrm{CO}_{2}$ em excesso foi absorvido com auxílio de uma solução de hidróxido de potássio a $40 \%$. O monitoramento da temperatura foi realizado diariamente, por meio de um termômetro inserido na polpa de um fruto, e o controle foi realizado por termostatos.

Os frutos, após os 57 dias de armazenamento e mais dois dias de exposição à temperatura de $20^{\circ} \mathrm{C}$, foram avaliados quanto à firmeza de polpa, aos sólidos solúveis (SS), à acidez titulável, à taxa respiratória, à síntese de etileno, à atividade ACC oxidase e ocorrência 
de podridões, ao escurecimento interno e externo e à lanosidade. A firmeza de polpa foi determinada na porção equatorial do fruto, com um penetrômetro manual, com uma ponteira de 7,9mm de diâmetro. Os sólidos solúveis foram determinados com o auxílio de um refratômetro manual com correção da temperatura. A acidez titulável foi determinada por meio da titulação de $10 \mathrm{ml}$ de suco diluídos em $100 \mathrm{ml}$ de água destilada, com uma solução de $\mathrm{NaOH}$ 0,1N até pH 8,1.

A avaliação da ocorrência de podridões foi realizada pela contagem de frutos com lesões podres, sendo os resultados expressos em percentagem. $\mathrm{O}$ escurecimento externo foi expresso por índice de 0-4, em que foi considerado índice $0=$ sem escurecimento, índice $1=$ frutos com até $10 \%$ da epiderme escurecida, índice $2=10-30 \%$ da epiderme escurecida, índice 3 =frutos com $30-50 \%$ de escurecimento na epiderme e índice $4=$ com mais de $50 \%$ de escurecimento. O escurecimento interno foi determinado visualmente e expresso em percentagem de frutos com polpa escurecida. A lanosidade foi determinada de forma subjetiva por meio da pressão do fruto com os dedos e observação visual da presença ou ausência de suco e quantificada por um índice de 1 a 3, de acordo com o volume de suco extraído, sendo índice $1=$ fruto não lanoso, índice 2 =fruto mediamente lanoso e índice $3=$ fruto totalmente lanoso. O índice de cada unidade experimental foi obtido multiplicando-se o número de frutos pelo nível constatado, cujo somatório foi dividido pelo número total de frutos da amostra.

A produção de etileno foi determinada no primeiro e no segundo dia de exposição dos frutos a $20^{\circ} \mathrm{C}$, com a utilização de aproximadamente $1.400 \mathrm{~g}$ de frutos, acondicionados em recipientes com volume de 5.000mL. Estes foram fechados hermeticamente durante aproximadamente uma hora. Para a análise da produção de etileno, foram injetadas duas amostras de $1 \mathrm{~mL}$ de gás, provenientes de cada recipiente, em um cromatógrafo a gás, marca Varian, equipado com um detector de ionização por chama (FID) e coluna Porapak N80/100. Calculou-se a síntese de etileno em $\mu \mathrm{L} \mathrm{C}_{2} \mathrm{H}_{4}$ $\mathrm{kg}^{-1} \mathrm{~h}^{-1}$ pela concentração de etileno, da massa dos frutos, do volume do espaço livre no recipiente e do tempo de fechamento. A respiração foi determinada pela quantificação da produção de $\mathrm{CO}_{2}$. $\mathrm{O}$ ar do mesmo recipiente utilizado para a determinação do etileno foi circulado por meio de um analisador eletrônico de $\mathrm{CO}_{2}$, marca Agri-datalog. A partir da concentração de $\mathrm{CO}_{2}$, do espaço livre do recipiente, do peso do fruto e do tempo de fechamento, foi calculada a respiração em $\mathrm{mL}$ $\mathrm{CO}_{2} \mathrm{~kg}^{-1} \mathrm{~h}^{-1}$.

A avaliação da atividade da enzima ACC (ácido 1-carboxílico-1-aminociclopropano) oxidase foi realizada de acordo com a metodologia proposta por BUFLER (1986). Foram retiradas algumas amostras de casca da região equatorial dos frutos, totalizando $3 g$. Estas foram imersas em solução contendo $0,1 \mathrm{mM}$ de ACC (ácido 1-carboxílico-1-aminociclopropano), em $10 \mathrm{mM}$ do tampão MES (ácido 2 (N-morfolino) etanossulfônico), em pH 6,0. Após 30 minutos, as amostras foram acondicionadas em seringas herméticas de $50 \mathrm{~mL}$, adicionando-se $1 \mathrm{~mL}$ de $\mathrm{CO}_{2}$ e, após de 30 minutos, realizou-se a determinação da concentração de etileno nas seringas, sendo os dados expressos em $\mu \mathrm{L} \mathrm{C}_{2} \mathrm{H}_{4} \mathrm{~kg}^{-1} \mathrm{~h}^{-1}$.

Os dados foram submetidos à análise de variância. Os dados expressos em percentagem foram transformados para arco seno $\sqrt{\mathrm{x} / 100}$, para posterior análise da variância. As médias foram comparadas pelo teste de Tukey, em nível de 5\% de probabilidade de erro.

\section{RESULTADOS E DISCUSSÃO}

Após 57 dias de armazenamento a $-0,5^{\circ} \mathrm{C}$ mais dois dias de exposição dos frutos à temperatura de $20^{\circ} \mathrm{C}$, observou-se menor incidência de podridões no tratamento com atmosfera refrigerada, não diferindo do tratamento com pressão parcial de $2,0 \mathrm{kPa}$ de $\mathrm{O}_{2}+$ $8 \mathrm{kPa}$ de $\mathrm{CO}_{2}$ (Figura 1). O crescimento de fungos, em frutos armazenados, pode ser reduzido por meio do abaixamento da temperatura e pelo aumento da pressão parcial de CO (AGAR et al., 1990). Nesse sentido, NAVA \& BRAĆCKMANN, (2002) encontraram menor incidência de podridões em pêssegos 'Chiripá' armazenados sob 2,0kPa de $\mathrm{O}_{2}+8,0 \mathrm{kPa}$ de $\mathrm{CO}_{2}$.

Com relação ao escurecimento externo, os frutos armazenados sob pressão parcial de $2,0 \mathrm{kPa}$ de $\mathrm{O}_{2}+8,0 \mathrm{kPa}$ de $\mathrm{CO}_{2}$ apresentaram menor incidência, diferindo apenas do tratamento com $1,0 \mathrm{kPa}$ de $\mathrm{O}_{2}+$ $3 \mathrm{kPa}$ de $\mathrm{CO}_{2}$ (Figura1).

Quando avaliados os parâmetros de qualidade responsáveis pelo sabor dos frutos, o sólidos solúveis (SS) não foram influenciados pelas diferentes condições de atmosfera controla (Figura 1), enquanto que a acidez titulável foi maior em atmosfera controlada (Figura 1). NAVA (2001) também obteve acidez mais elevada em pêssegos 'Chiripá' armazenados em atmosfera controlada em comparação com os frutos armazenados em atmosfera refrigerada. Ao avaliar as características fisiológicas dos frutos, observou-se que o escurecimento interno da polpa foi maior em condições de atmosfera refrigerada (Figura 1). De acordo com KADER (1986), frutas em AC apresentaram menor escurecimento que em AR, provavelmente, devido à redução das concentrações de $\mathrm{O}_{2}$ e aumento

Ciência Rural, v.39, n.7, out, 2009. 


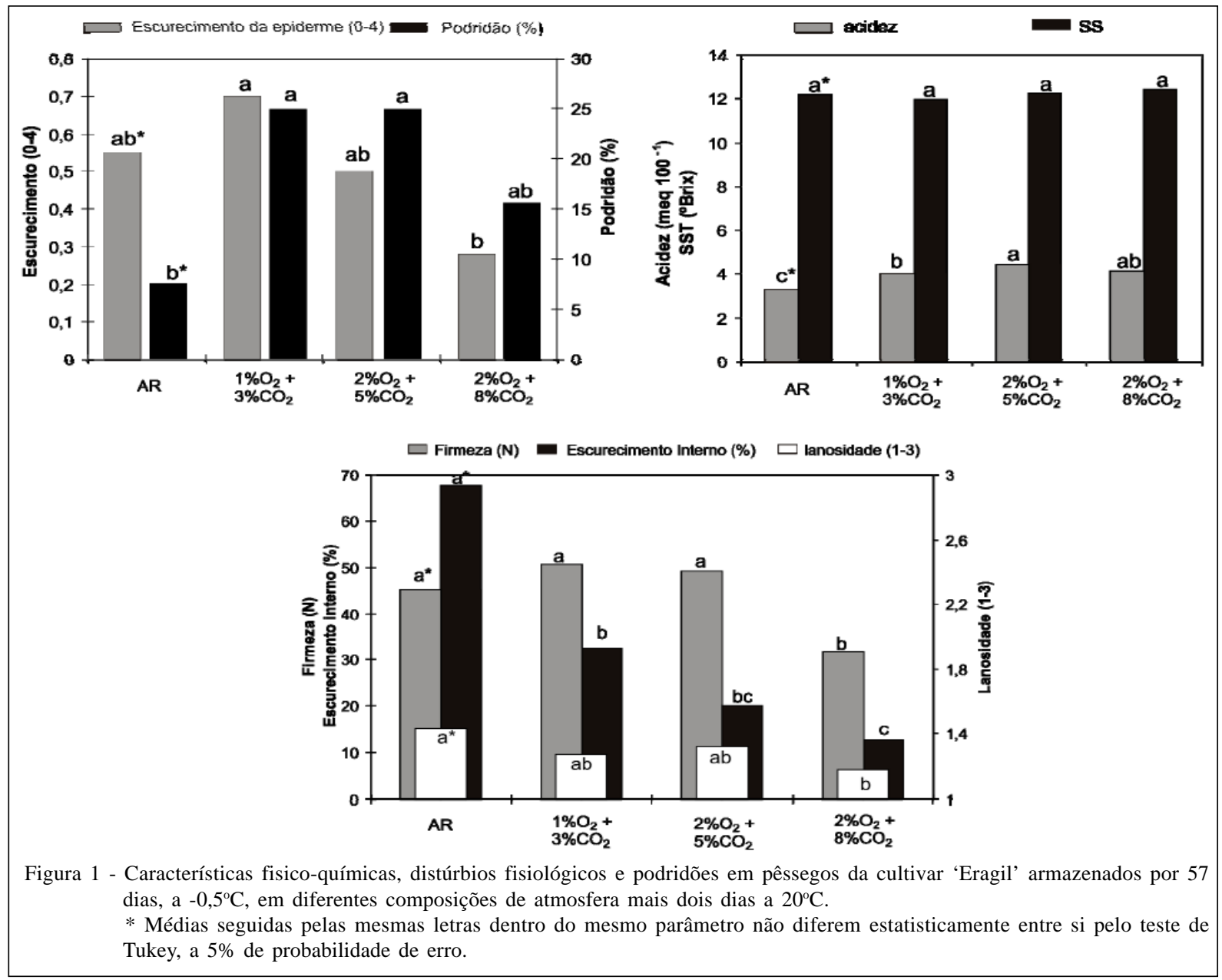

do $\mathrm{CO}_{2}$, combinado com a temperatura de $-0,5^{\circ} \mathrm{C}$, que diminuem os processos metabólicos, provocando menor escurecimento. A firmeza de polpa foi menor nos frutos submetidos ao tratamento com pressão parcial de 2,0kPa de $\mathrm{O}_{2}+8,0 \mathrm{kPa}$ de $\mathrm{CO}_{2}$ (Figura 1). Esse resultado reforça a teoria de LURIE (1992), o qual afirma que os frutos armazenados em alto $\mathrm{CO}_{2}$ mantêm a habilidade de amadurecer durante a vida de prateleira sem apresentar distúrbios, como a lanosidade.

A taxa respiratória dos frutos não diferiu estatisticamente entre os tratamentos (Figura 2). A síntese de etileno, tanto na saída da câmara, quanto aos dois dias, e a atividade da ACC-oxidase foram menores no tratamento sob atmosfera refrigerada. O uso de altos níveis de $\mathrm{CO}_{2}$ pode inibir vários processos metabólicos dependentes de etileno, como a produção autocatalítica de etileno, portanto pode atrasar o amadurecimento de frutos climatéricos (MATHOOKO, 1996). Assim, durante o armazenamento em AC, os altos níveis de $\mathrm{CO}_{2}$ e baixos de $\mathrm{O}_{2}$ reduzem a síntese de etileno, possivelmente pela inibição da ACC sintase e
ACC oxidase, respectivamente. Entretanto, GIEHL, et al. (2008) afirmaram que, quando os frutos passam da condição de atmosfera controlada para as condições ambientais, a produção de etileno aumenta, superando a produção de etileno de frutos armazenados em atmosfera refrigerada. A AC causa um aumento na concentração de ACC e esta é convertida em etileno após o aumento da temperatura dos frutos, justificando os resultados obtidos no presente trabalho, em que, provavelmente, essa maior produção de etileno em condições de AC está relacionada à alta atividade da ACC oxidase (Figura 2).

Quando avaliada a incidência de frutos lanosos, constatou-se que o índice de lanosidade diferiu estatisticamente somente entre os frutos sob atmosfera refrigerada e os submetidos à pressão parcial de 2,0kPa de $\mathrm{O}_{2}+8,0 \mathrm{kPa}$ de $\mathrm{CO}_{2}$, em que se obteve sua menor incidência (Figura 1). Segundo LURIE (1992), a atmosfera com alto $\mathrm{CO}_{2}$ mantém a habilidade dos frutos, armazenados sob temperaturas críticas, em amadurecer normalmente sem a ocorrência significativa de 

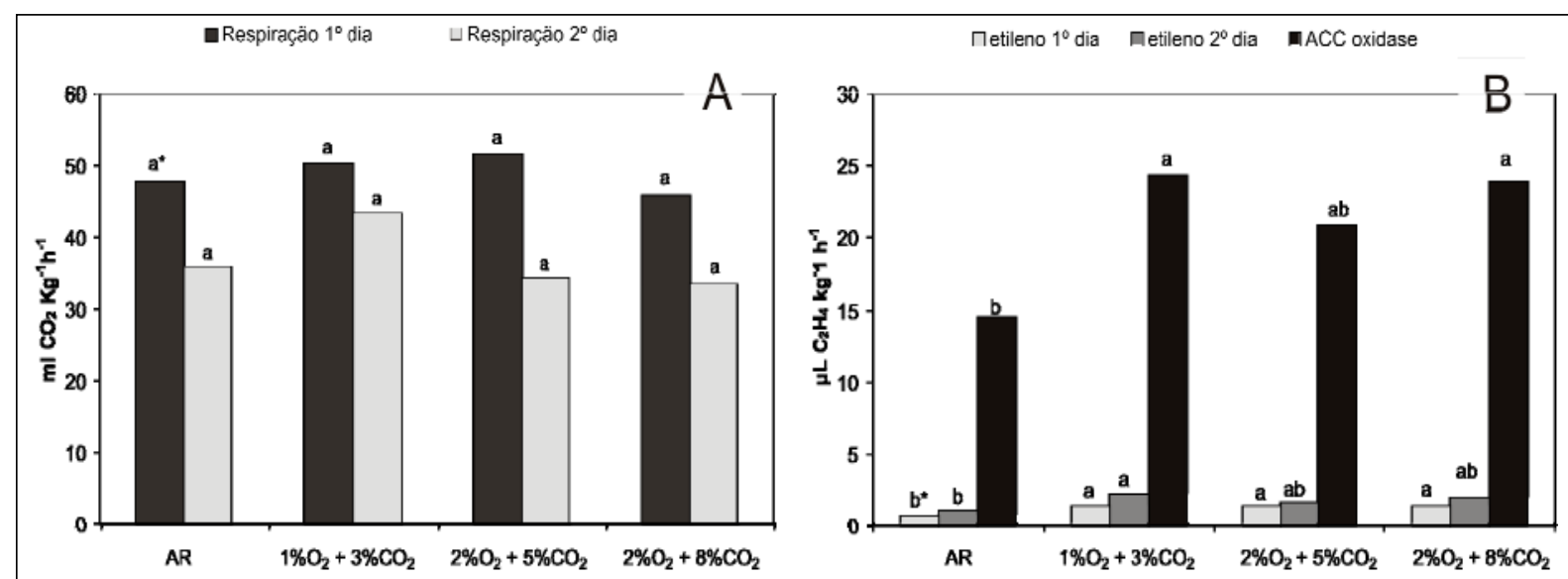

Figura 2 - Taxa respiratória (A), produção de etileno (B) e atividade ACC oxidase (B) em pêssegos da cultivar 'Eragil' armazenados por 57 dias, a $-0,5^{\circ} \mathrm{C}$, em diferentes composições de atmosfera mais dois dias a $20^{\circ} \mathrm{C}$.

* Médias seguidas pelas mesmas letras dentro do mesmo parâmetro não diferem estatisticamente entre si pelo teste de Tukey, a 5\% de probabilidade de erro.

distúrbios fisiológicos. Além disso, esse tratamento apresentou uma elevada atividade ACC oxidase, o que resultou em um restabelecimento da produção de etileno, contribuindo para a redução na ocorrência de lanosidade. Esses resultados estão de acordo com GIEHL, et al. (2008), os quais constataram que a presença do etileno exógeno ou endógeno durante o armazenamento ou a habilidade dos frutos em produzir etileno após o armazenamento reduz a ocorrência de lanosidade e aumenta a capacidade de restabelecimento da suculência nos frutos durante o período de prateleira.

\section{CONCLUSÕES}

A condição de atmosfera controlada mais eficiente para o armazenamento de pêssegos 'Eragil' é 2,0kPa de $\mathrm{O}_{2}+8,0 \mathrm{kPa}$ de $\mathrm{CO}_{2}$ que permitiu a manutenção da qualidade dos frutos, diminuindo a incidência de lanosidade e a ocorrência de escurecimento interno da polpa. Além disso, essa condição permite o amadurecimento normal do fruto, reduzindo os distúrbios fisiológicos durante a vida de prateleira.

\section{REFERÊNCIAS}

AGAR, T. et al. Effect of high $\mathrm{CO}_{2}$ and low $\mathrm{O}_{2}$ concentrations on the growth of Botrytis cinerea at different temperatures. Gartenbauwissenschaft, Berlin, v.55, n.5, p.219-222, 1990.

BEAUDRY, R.M. Effect of $\mathrm{O}_{2}$ and $\mathrm{CO}_{2}$ partial pressure on selected phenomena affecting fruit and vegetable quality. Postharvest Biology and Technology, Amsterdam, v.15, p.293-303, 1999. Disponível em: <http://www.sciencedirect.com/ science?_ob=ArticleURL\&_udi=B6TBJ-3W07NN1C \&_user $=687358$ \&_cover D a t e $=03 \% 2$ F $31 \% 2$ F1999\&_rdoc $=11 \&$ \&mt=high\&_orig=browse\&_srch=doc-
info(\%23toc\%235144\%231999\%23999849996\%2375031\%23FLA \%23display\%23Volume)\&_cdi=5144\&_sort=d\&_docanchor $=$ \&_ct $=16 \&$ \&_acct $=$ C $000037899 \&$ \&_version $=1 \&$ \&_url V ersion=0\&_userid=687358\&md5=9b73bd78f5f16a13011d9ddaba26d64d $>$. Acesso em: 13 de maio de 2009. doi: 10.1016/S09255214(98)00092-1.

BRACKMANN, A. et al. Armazenamento de pêssegos cv. 'Granada' em atmosfera controlada, visando ao transporte a longas distâncias. Ciência Rural, Santa Maria, v.37, n.3, p.676-681, 2007. Disponível em: <http://www.scielo.br/ s cielo.php? script =s ci_art text \& pid=S 0103 $84782007000300012 \& \operatorname{lng}=\mathrm{en} \& \mathrm{nrm}=\mathrm{iso} \& \mathrm{t} \operatorname{lng}=\mathrm{pt}>$. Acesso em: 13 de maio de 2009 . doi: 10.1590/S010384782007000300012 .

BRACKMANN, A.; CHITARRA, A.B. Atmosfera controlada e atmosfera modificada. In: Armazenamento e processamento de produtos agrícolas. Lavras: UFLA/ SBEA, 1998. p.133-170.

BUFLER, G. Ethylene-promoted conversion of 1aminocyclopropene-1-carboxylic acid to ethylene in peel of apple at various stages of fruit development. Plant Physiology, Rockville, v.80, p.539-543, 1986.

CERETTA, M. et al. Conservação em atmosfera controlada de pêssego cultivar Eldorado. Ciência Rural, Santa Maria, v.30, n.1, p.73-79, 2000. Disponível em: <http://www.scielo.br/ scielo.php? script = s ci_art text \& pid=S 0103 $84782000000100012 \& \operatorname{lng}=\mathrm{en} \& \mathrm{nrm}=\mathrm{iso} \& \mathrm{t} \operatorname{lng}=\mathrm{pt}>$. Acesso em: 13 de maio de 2009. doi: 10.1590/S010384782000000100012 .

GIEHL, R.F.H. et al. CA affects juiciness in peaches due to an earlier post-storage ethylene production. Acta Horticulturae, Weingarten, Germany. n.796, 185-189, 2008.

HADLICH, E.; ARAÚJO, P.J. Frigoconservação continua e intermitente de pêssegos (Prunus persica L.) cultivares 'Diamante' e 'Eldorado'. Revista Brasileira de Fruticultura, Cruz das Almas, v.15, n.1, p.177-183, 1993.

Ciência Rural, v.39, n.7, out, 2009. 
KADER, A.A. Biochemical and physiological basis for effects of controlled and modified atmospheres on fruits and vegetables. Food Technology, Chicago, v.40, n.5, p.99-104, 1986.

KADER, A.A.; MITCHELL, F.G. Maturity and quality. In: Peaches, plums and nectarines-growing and handling for fresh market. Oakland: Univ. Calif. Div. Agric. and Natural Resources, 1989. p.191-196.

KENDE, H. Ethylene biosynthesis. Annual Review of Plant Physiology and Molecular Biology, Palo Alto, v.44, p.283307, 1993. Disponível em: <http://arjournals.annualreviews.org/ doi/abs/10.1146/annurev.pp.44.060193.001435?prevSearch=\% 5Bauthor\%3A+Kende\%2C+H\%5D\&searchHistoryKey=> . Acesso em: 13 de maio de 2009. doi: 10.1146/ annurev.pp.44.060193.001435.

LURIE, S. Controlled atmosphere storage to prevent physiological disorders in nectarines. International Journal of Food Science and Technology, v.27, p.507-514, 1992.

LURIE, S.; CRISOSTO, C.H. Chilling injury in peach and nectarine. Postharvest Biology and Technology, Amsterdam, v.37, n. 3, p.195-208, 2005. Disponível em: <http:// www.sciencedirect.com/science?_ob=ArticleURL\&_udi=B6TBJ$4 \mathrm{GPW} 6 \mathrm{GP}-1 \&$ us er $=687358 \&$ coverDate $=09 \% 2 \mathrm{~F}$ $30 \% 2 F 2005 \& \_r d o c=2 \& \_f m t=h i g h \& \_$orig $=$browse\&_srch $=$doc info(\%23toc\%235144\%232005\%23999629996\%23604128\%23FL A\%23display\%23Volume)\&_cdi $=5144 \& \_s o r t=d \& \_d o c a n c h o r=\&$ _ct $=13 \&$ \&_acct $=$ C $000037899 \&$ \& version $=1 \&$ _urlVersio $\mathrm{n}=0$ \&_userid=687358\&md5=929b692da6c0e26fb30ca1c60fe498a0>. Acesso em: 13 de maio de 2009. doi: 10.1016/ j.postharvbio.2005.04.012.

MATHOOKO, F.M. Regulation of genes encoding ethylene biosynthetic enzymes in peach (Prunus persica L.) fruit by carbon dioxide and 1methylcyclopropene. Postharvest Biology and Technology, Amsterdam, v.21, n.3, p.265-281, 2001. Disponível em: <http:// www.sciencedirect.com/science? ob $=$ ArticleURL\&_udi=B6TBJ42 D 2 B V $6-2 \&$ \& user $=687358 \&$ \& coverDat e $=02 \% 2$ F28\%2F2001\&_rdoc $=2 \& \_f m t=h i g h \& \_o r i g=b r o w s e \& \_s r c h=d o c-$ info(\%23toc\%235144\%232001\%23999789996\%23229139\%2 3FLA\%23display\%23Volume)\&_cdi=5144\&_sort=d\&_docanchor=\& _ $\mathrm{ct}=13 \&$ \&_acct $=\mathrm{C} 000037899 \&$ _version $=1 \&$ \&_url Version=0\&_userid=687358\&md5=2e598091ef53378acd55e8b4c4287267>. Acesso em: 13 de maio de 2009. doi: 10.1016/S09255214(00)00158-7.
MATHOOKO, F.M. Regulation of respiratory metabolism in fruits and vegetables by carbon dioxide. Postharvest Biology and Technology, Amsterdam, v.9, p.247-264, 1996. Disponível em: <http:// www.sciencedirect.com/science?_ob=ArticleURL\&_udi=B6TBJ3RG551D-W \& user $=687358 \&$ coverDate $=12 \% 2$ $\mathrm{F} 31 \% 2 \mathrm{~F} 1996 \&$ rdoc $=1 \&$ fmt $=$ high\&_orig $=$ browse\&_srch $=$ docinfo(\%23toc\%235144\%231996\%23999909996\%23588313 \%23FLP\%23display\%23Volume)\&_cdi=5144\&_sort=d\&_docan chor $=\& \quad c t=15 \&$ a c $\mathrm{ct}=\mathrm{C} 000037899 \&$ version $=1$ \&_urlVersion=0\&_userid=687358\&md5=15aa06835a2a448cfe962ale966b9444>. Acesso em: 13 de maio de 2009. doi: 10.1016/S09255214(96)00019-1.

NAVA, G.A. Efeito da atmosfera controlada, eliminação do etileno da câmara e do pré-resfriamento sobre a qualidade de pêssegos, cv. Chiripá. 2001. 73f. Dissertação (Mestrado em Agronomia) - Universidade Federal de Santa Maria, Santa Maria.

NAVA, G.A.; BRACKMANN, A. Armazenamento de pêssegos (Prunus persica (L.) Batsch), cv. 'Chiripá', em atmosfera controlada. Revista Brasileira de Fruticultura, Jaboticabal, v.24, n.2, p.328-332, 2002. Disponível em: <http:// www.scielo.br/scielo.php?script=sci_arttext\&pid=S0100$29452002000200011 \& \operatorname{lng}=p t \& n r m=i$ so $\&$ tlng $=p t>$. Acesso em: 13 de maio de 2009. doi: 10.1590/S010029452002000200011.

SAQUET, A.A. et al. Changes in ATP, ADP and pyridine nucleotide levels related to the incidence of physiological disorders in 'Conference' pears and 'Jonagold' apples during controlled atmosphere storage. Journal of Horticultural Science \& Biotechnology, Ashford, v.75, p.243-249, 2000.

WATADA, A.E. et al. Sensory compositional, and volatile attributes of controlled atmosphere stored peaches. Journal of the American Society for Horticultural Science, Alexandria, v.104, n.5, p.626-629, 1979. Disponível em: $<\mathrm{ht} \mathrm{t}$ p:// w w w. sc i e n c ed i rect.com/ science? ob=ArticleURL\&_udi=B6TBJ-3RG551DW \&_us e r $=687358 \&$ _ c over D a t e $=12 \% 2$ F 31 \%2F1996\&_rdoc $=1 \&$ \& fmt=high\&_orig=browse\&_srch $=$ docinfo(\%23toc\%235144\%231996\%23999909996\%23588313\%2 3FLP\%23display\%23Volume)\& cdi $=5144 \&$ sort $=\mathrm{d} \&$ do canchor $=$ \&_ct $=15 \&$ \&_act $=C 000037899 \&$ \& version $=1 \&$ _ urlVersion $=0$ \& userid $=687358 \&$ md5 $=15$ aa06835a2a448cfe962a1e966b9444 $>$. Acesso em: 13 de maio de 2009. doi: 10.1016/S09255214(96)00019-1. 